\title{
Addiction Training: Striving to Fill an Unmet Need
}

\author{
Smita Das ${ }^{1} \cdot$ Laura Weiss Roberts ${ }^{2}$ (D)
}

Received: 4 March 2016 / Accepted: 14 March 2016/Published online: 28 March 2016

(C) Academic Psychiatry 2016

The health consequences of addiction are immense, and the need for specialized addiction treatment far exceeds therapeutic resources that currently exist. Clinicians across the spectrum of health care struggle to address or treat disorders related to use or misuse of opiates, alcohol, tobacco, stimulants, marijuana, and prescription medications. In a recent national survey, an estimated 27 million Americans aged 12 years or older (10.2\% of the population) had used an illicit drug in the past month, and yet only $11 \%$ receive treatment at a specialty facility [1]. Moreover, abuse of tobacco, alcohol, and illicit drugs is costly to the USA, resulting in more than $\$ 700$ billion annually in costs related to crime, lost work productivity, and health care [2]. Internationally, the World Health Organization reports that in 2012 about 3.3 million net deaths, or $5.9 \%$ of all global deaths, were attributable to alcohol consumption [3]. Tobacco, the leading cause of preventable death in the USA, also has international impact, killing around 6 million people each year [4].

With the passage of the Affordable Care Act and the Mental Health Parity and Addiction Equity Act in the USA, insurance coverage for substance use disorders has increased. All insurers, including Medicaid, are now required to cover the treatment of drug and alcohol addiction. However, there remains a deficit of providers. A recent survey integrating national data on substance use disorders (SUDs) and practi-

Laura Weiss Roberts

LWRoberts.EIC@gmail.com

1 University of California San Francisco, San Francisco, CA, USA

2 Stanford University, Stanford, CA, USA tioners found that the average number of advanced providers (counselors, school psychologists, psychiatrists, social workers) per 1000 patients with SUDs in the USA is 32, ranging from 11 in Nevada to 70 in Vermont [5]. It is important to note that these practitioners are not specifically or necessarily physicians. Indeed, for a country of over 320 million people, there are only about 2000 board-certified addiction psychiatrists and about 2500 physicians certified in addiction medicine [6].

Because of the small number of specialists relative to the extraordinary level of societal need, the integration of addiction-related curricular topics into medical education and psychiatry residencies is critical. We are pleased to present a collection of papers on addiction training that came to our journal Academic Psychiatry recently, highlighting the importance of advancing education in this subspecialty field. The collection offers valuable insights into how to enhance addiction training as relevant to academic psychiatrists.

Addiction training can be effective and useful in all levels of education: undergraduate medical education, residency, and postgraduate training. At the undergraduate medical education level, addiction training is absolutely necessary given the high prevalence of substance use. Medical students entering any field of medicine are likely to encounter addiction, which can affect their treatment: surgeons will consider addiction pre-operatively, gastroenterologists will face liver consequences, cardiologists will see the effects of tobacco, all specialties will feel the impact of opiates, and the list continues. Addiction also offers an ideal platform for teaching behavior change. Framing treatment in behavior change can help medical students offer the same strategies to patients in any field. For example, motivational interviewing or the transtheoretical stages of change can help patients with other health concerns like diabetes management, obesity, medication compliance, and so on. 
Articles in this issue draw attention to innovative integration of addiction education into undergraduate medical curricula. Ram and Chisolm [7] comment on the deficiency, need, and opportunity to integrate addiction training into medical schools, while Kastenholz and Agarwal [8] highlight reflections of medical students attending Alcoholics Anonymous meetings. Behavioral Drug and HIV Risk Reduction Counseling in low- and middle-income countries is discussed by Marienfield and Charwaski [9], underscoring the impact of addictions internationally and across disciplines.

In reference to psychiatry residencies, the Accreditation Council for Graduate Medical Education (ACGME) currently only requires 1 month of addiction treatment experience out of 48 months of training [10]. At the same time, addiction is the leading cause of death among both psychiatric patients and the US population in general [11]. Approximately 8.9 million adults have co-occurring disorders (both a mental and substance use disorder). Only $7.4 \%$ of those individuals receive treatment for both conditions, with $55.8 \%$ receiving no treatment at all [12]. With such a strong prevalence in our patients, it is crucial that residencies seek to provide training beyond that which is minimally required.

The lack of priority of addiction training throughout medicine may be a reflection of the stigma associated with substance use and insufficient appreciation of the recovery movement in the context of addiction. Avery, Zerbo, and Ross [13] discuss stigmatization and negative attitudes toward the field by psychiatrists and offer strategies to improve those attitudes. Another innovative way to improve understanding of addictions is offered by Agrawal et al. [14] as they describe how service users (patients and caregivers) can advise senior psychiatry residents. In the same vein of recovery-oriented approaches, two articles discuss this transformation in training and attitudes. Sowers et al. [15] describe recovery-oriented curricula and modules. Gambino, Pavlo, and Ross [16] offer psychiatry trainee reflections on what it means to engage in recovery-oriented care, using the Substance Abuse and Mental Health Services Administration definitions.

Training in specialties like psychiatry that manage a greater volume of patients with substance use disorders can offer a wealth of evidence-based strategies and allow for an interdisciplinary breadth of training. Substance use disorders training ranges in areas from medication to therapy to complementary approaches. In the area of medications, Suzuki et al. [17] note that buprenorphine waiver trainings are increasingly encouraged in psychiatry residency programs and those not currently offering them are eager to integrate, especially given the prevalence of opiate use disorders and strong evidence base behind buprenorphine treatment. Therapy is integral to treatment of addictions; Sanchez-Ramirez et al. [18] discuss training senior residents in addiction through an intensive outpatient program. Complementary techniques in addiction are highlighted in an article on acupuncture by Serafini et al. [19]. Also important is the interdisciplinary role that addiction psychiatrists have, which is highlighted within the Edens et al. [20] brief report on the overlap of chronic pain and addiction.

This collection highlights findings from advanced levels of training such as addiction psychiatry fellowship programs; however, given the demand for addiction treatment, more specialty providers are needed. Distinctive in addiction treatment is the multidisciplinary nature of the field - in addition to an addiction psychiatry ACGME-accredited fellowship, psychiatrists and non-psychiatrists can pursue addiction medicine by becoming certified by the American Board of Addiction Medicine. Levounis, Zerbo, and Aggarwal [21] emphasize innovative ways to incorporate flexible addiction education into psychiatric and non-psychiatric training to meet the demands of a growing patient population. Finally, it is important to encourage sustainability in addiction education and workforce; Muvvala et al. [22] discuss innovative ways to use case conferences to educate future educators in addiction psychiatry.

We hope that the readers of Academic Psychiatry will find this collection to have value in presenting state-of-the-art and practical ways for academicians to enhance addiction training across disciplines and levels of education. We extend our special thanks to the authors of the articles included in this collection, as they work every day to help fill the gap in treatment through their practice and academic work. Substance use disorders unfortunately continue to grow and affect patients in direct and indirect ways, often greatly shaping their lives. Reducing stigma, enhancing awareness, and promoting confidence in addiction treatment with psychiatrists and allied health professionals is imperative to the health of our patients.

\section{Compliance with Ethical Standards}

Disclosure On behalf of all authors, the corresponding author states that there are no conflicts of interest.

\section{References}

1. Substance Abuse and Mental Health Services Administration. Behavioral health trends in the United States: results from the 2014 National Survey on Drug Use and Health. 2015. http:// www.samhsa.gov/data/sites/default/files/NSDUH-FRR1-2014/ NSDUH-FRR1-2014.pdf. Accessed 29 Feb 2016.

2. National Institute on Drug Abuse. Drugs, brains, and behavior: The science of addiction. 2014. http://www.drugabuse.gov/ publications/science-addiction/introduction. Accessed 29 Feb 2016.

3. World Health Organization. Global status report on alcohol and health. 2014. http://www.who.int/substance abuse/publications/ global_alcohol_report/msb_gsr_2014_1.pdf?ua=1. Accessed 29 Feb 2016.

4. World Health Organization. WHO report on the global tobacco epidemic, 2011: warning about the dangers of tobacco. 2011. 
http://apps.who.int/iris/bitstream/10665/44616/1/9789240687813 eng.pdf. Accessed 29 Feb 2016.

5. Advocates for Human Potential, Inc. The adequacy of the behavioral health workforce to meet the need for services: overview of key findings. 2014. http://www.ahpnet.com/Files/AHP-BHWorkforce-Paper-July-2014.aspx. Accessed 29 Feb 2016.

6. American Society of Addiction Medicine. Public resources. 2016. http://www.asam.org/for-the-public/what-is-an-addictionspecialist. Accessed 29 Feb 2016.

7. Ram A, Chisolm MS. The time is sow: improving substance abuse training in medical schools. Acad Psychiatry. 2016; 40(3).

8. Kastenholz KJ, Agarwal G. A qualitative analysis of medical students' reflection on attending an Alcoholics Anonymous meeting: insights for future addiction curricula. Acad Psychiatry. 2016; 40(3).

9. Marienfeld C, Chawarski M. Teaching psychosocial interventions for opioid use disorder in low and middle income countries: Malaysia and China. Acad Psychiatry. 2016; 40(3).

10. Accreditation Council for Graduate Medical Education. ACGME program requirements for graduate medical education in psychiatry (effective July 2014). 2015. https://www.acgme.org/acgmeweb/ portals/0/pfassets/programrequirements/400_psychiatry_ 07012014.pdf. Accessed 29 Feb 2016.

11. U.S. Department of Health and Human Services. The health consequences of smoking - 50 years of progress: a report of the Surgeon General, 2014. U.S. Department of Health and Human Services. Rockville, MD; 2014.

12. Substance Abuse and Mental Health Services Administration, Office of Applied Studies. Results from the 2009 National Survey on Drug Use and Health. U.S. Department of Health and Human Services. Rockville, MD; 2010.
13. Avery J, Zerbo E, Ross S. Improving psychiatrists' attitudes towards individuals with psychotic disorders and co-occurring substance use disorders. Acad Psychiatry. 2016; 40(3).

14. Agrawal S, Capponi P, Kidd S, et al. From surviving to advising: a novel course pairing mental health and addictions service users as advisors to senior psychiatry residents. Acad Psychiatry. 2016; 40(3).

15. Sowers W, Primm A, Cohen D, Pettis J, Thompson K. Transforming psychiatry: a curriculum on recovery-oriented care. Acad Psychiatry. 2016; 40(3).

16. Gambino M, Pavlo A, Ross DA. Recovery in mind: perspective from postgraduate psychiatric trainees. Acad Psychiatry. 2016; 40(3).

17. Suzuki J, Ellison TV, Connery HS, Surber C, Renner JA. Training in buprenorphine and office-based opioid treatment: a survey of psychiatry residency training programs. Acad Psychiatry. 2016; 40(3).

18. Sanchez-Ramirez JP, Gakhal R, Oakmas SA. Addiction psychiatry in PGY-3; use of the intensive outpatient treatment setting to train senior residents. Acad Psychiatry. 2016; 40(3).

19. Serafini K, Bryant K, Ikomi J, LaPaglia D. Training psychiatry addiction fellows in acupuncture. Acad Psychiatry. 2016; 40(3).

20. Edens EL, Gafni I, Encandela J. Addiction and chronic pain: training addiction psychiatrists. Acad Psychiatry. 2016; 40(3).

21. Levounis P, Zerbo E, Aggarwal R. A "middle way": introducing a flexible year-long program to prepare for certification in addiction medicine. Acad Psychiatry. 2016; 40(3).

22. Muvvala SB, Marienfeld C, Encandela J, Petrakis I, Edens EL. An innovative use of case conference to teach future educators in addition psychiatry. Acad Psychiatry. 2016; 40(3). 